\title{
Spontaneous Uterine Rupture at 15 Weeks' Gestation in a Patient with a History of Cesarean Delivery after Removal of Shirodkar Cerclage
}

\author{
Serika Kanao, MD ${ }^{1}$ Aya Fukuda, $\mathrm{MD}^{1}$ Hirotsugu Fukuda, MD ${ }^{1}$ Mayuko Miyamoto, MD ${ }^{1}$ \\ Eriko Marumoto, $\mathrm{MD}^{1}$ Kiichiro Furuya, $\mathrm{MD}^{1}$ Rie Nishiyama, $\mathrm{MD}^{1}$ Chifumi Ohyagi, MD, $\mathrm{PhD}^{1}$ \\ Haruki Ogawa, MD, PhD ${ }^{1}$ \\ ${ }^{1}$ Department of Obstetrics and Gynecology, Osaka Kouseinenkin \\ Hospital, Osaka, Japan \\ Am J Perinatol Rep 2014;4:1-4. \\ Address for correspondence Aya Fukuda, MD, Department of \\ Obstetrics and Gynecology, Osaka Kouseinenkin Hospital, 4-2-78 \\ Fukushima Fukushima-ku, Osaka city, Osaka 553-0003, Japan \\ (e-mail: aya-swdn@okn.gr.jp).
}

\begin{abstract}
Keywords

- uterine rupture

- Shirodkar cerclage

- cesarean

- conization

- second trimester

A pregnant woman presented with acute upper abdominal pain and nausea at 15 weeks' gestation. She had a history of cesarean delivery for abruption after the removal of a Shirodkar cerclage that was placed because of cervical shortening caused by conization. She became pregnant again 14 months later. Ultrasonography revealed no significant findings, and a single intrauterine pregnancy with positive fetal heart activity was confirmed. An intestinal obstruction was suspected because abdominal radiography showed multiple air-fluid levels in the colon. Over the 3 hours following admission, her showed a large hemorrhage in the abdominal cavity, but the uterine wall appeared intact at this time. Subsequently, dynamic CT revealed discontinuity of the uterine muscle layer. During laparotomy, uterine rupture with complete opening of the uterine at the site of the previous transverse scar was identified. A dead fetus was located of packed red blood cells and 6 units of fresh frozen plasma for the resuscitation. She was discharged on the eighth postoperative day without any complications.
\end{abstract}

\section{Case Report}

A 31-year-old, gravida 4, para 2, woman presented with acute upper abdominal pain and nausea at 15 weeks' gestation. She had a history of appendectomy during childhood and a right ovarian cystectomy at 24 years of age. Her obstetrical history began with a full-term spontaneous vaginal delivery followed by conization because of cervical cancer at 28 years of age. Her second pregnancy ended in a missed abortion at 7 weeks' gestation with dilation and curettage treatment. In her previous pregnancy at 30 years of age, she received a historyindicated Shirodkar cerclage at 15 weeks' gestation. The cerclage was removed electively at 36 weeks' gestation. Six

received

August 13, 2013 accepted after revision September 16, 2013

published online

December 12, 2013 days after the release, she was admitted for unexpected massive bleeding because of cervical laceration at the 9 o'clock position before labor, and an emergency transverse cesarean delivery was performed under a diagnosis of abruption. Fourteen months after the cesarean section, she became pregnant again.

On admission, her general condition was quite stable. Her vital signs were as follows: pulse, $80 \mathrm{bpm}$; blood pressure, $108 / 70 \mathrm{~mm} \mathrm{Hg}$; and body temperature, $36.6^{\circ} \mathrm{C}$. On abdominal examination, she reported pain in the entire abdomen; the pain was strongest around the right hypochondriac region with rebound tenderness. Bowel sounds were audible but weak. Pelvic examination showed normal secretions, no
Copyright $\odot 2014$ by Thieme Medical Publishers, Inc., 333 Seventh Avenue, New York, NY 10001, USA. Tel: +1(212) 584-4662.
License terms

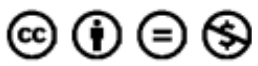

10.1055/s-0033-1358767. ISSN 2157-6998. 

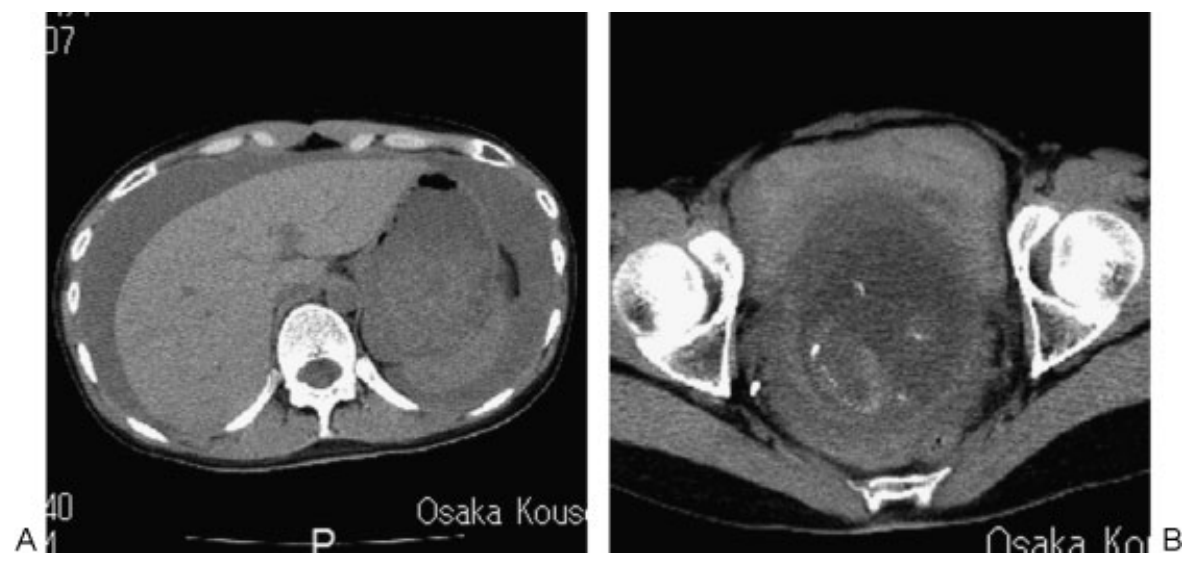

Fig. 1 (A) Plain computed tomography (CT) showing a large hemorrhage extending to occupy the perihepatic space in the abdominal cavity. (B) Plain CT showing continuity of the uterine muscle layer with an intrauterine fetus in the lower uterine segment.

vaginal bleeding, and no cervical dilation. Transvaginal ultrasonography revealed a shortened cervical length $(19 \mathrm{~mm})$ without funneling, no fluid collection in the cul-de-sac space, and a single intrauterine pregnancy with positive fetal heart activity. The placenta was located on the uterine fundus. Other organs, including the kidneys, gallbladder, and liver, were sonographically normal, and no fluid collection was detected in Morison's pouch or around the spleen. Laboratory tests performed at the time of admission did not show any significant findings, and hemoglobin level was $10.9 \mathrm{~g} / \mathrm{dL}$. Electrocardiography and chest radiography revealed no remarkable findings. Abdominal radiography showed multiple air-fluid levels in the colon without free air; therefore, an intestinal obstruction was suspected.

Over the 3 hours following admission, her symptoms gradually worsened and her hemoglobin level decreased to $7.9 \mathrm{~g} / \mathrm{dL}$; however, her vital signs remained stable. An addi-

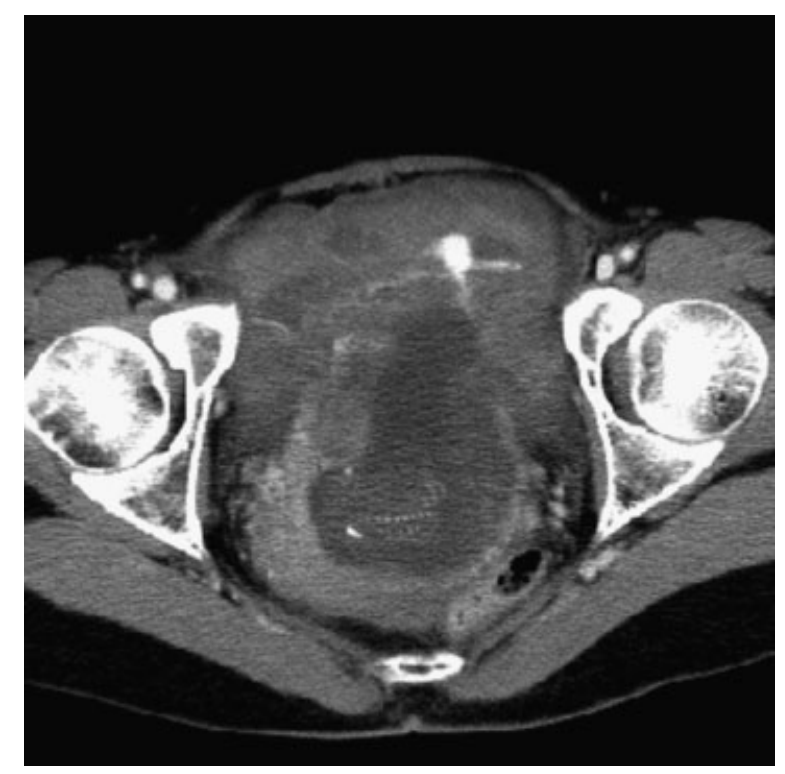

Fig. 2 Dynamic computed tomography revealing discontinuity of the uterine muscle layer in the lower uterine segment. tional ultrasonography examination showed a significant amount of fluid occupying the perihepatic space and a fetus with cardiac activity. A diagnostic plain abdominal computed tomography (CT) showed a large hemorrhage in the abdominal cavity ( - Fig. 1A), but the uterine wall appeared intact and intrauterine pregnancy was confirmed ( - Fig. 1B). An emergency laparotomy was indicated under a diagnosis of intraabdominal hemorrhage. The surgical department requested dynamic CT to determine the site of the incision. Dynamic CT is performed with rapid injection of contrast medium, usually with sequential scans at only one or a few levels; this modality is useful for screening patients with hemorrhage to detect the origin of the bleeding. Dynamic CT revealed discontinuity of the uterine muscle layer ( $\mathbf{- F i g . ~ 2 ) ; ~ t h e r e f o r e , ~ a ~ u t e r i n e ~}$ rupture was suspected.

While the patient was being prepared for emergency laparotomy, her blood pressure suddenly decreased to 78/ $51 \mathrm{~mm} \mathrm{Hg}$ and her heart rate increased. Therefore, aggressive resuscitation for hypovolemic shock was required. At the time of the operation, uterine rupture with complete opening of the uterine wall at the site of the previous transverse scar was found ( - Fig. 3). The fetus within the amniotic sac showed no cardiac activity and was located in a blood-filled abdominal cavity. The total amount of the hemorrhage in the abdominal cavity was approximately $3 \mathrm{~L}$. After the fetus and placenta were removed, the uterine scar was repaired using a doublelayer closure. During the preoperative, intraoperative, and postoperative periods, the patient received a total amount of 10 units of packed red blood cells and 6 units of fresh frozen plasma for the resuscitation. She recovered without any complications and was discharged on the eighth postoperative day.

\section{Discussion}

Among the most serious obstetric complications, uterine rupture is a potentially life-threatening condition for both the mother and the fetus. The representative risk factor is uterine scarring, mostly resulting from cesarean section, and the incidence may vary depending on the type of previous 


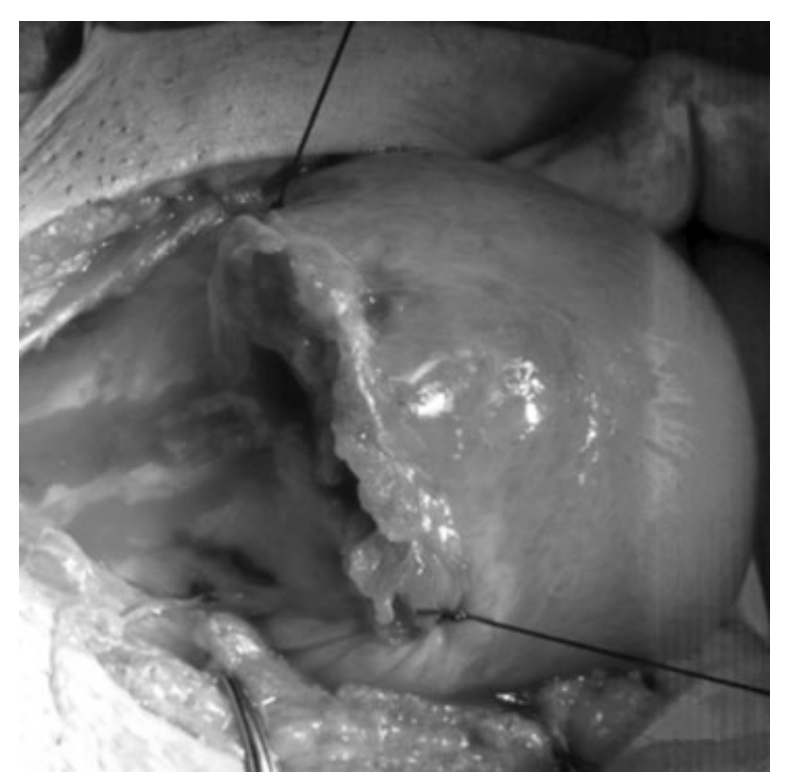

Fig. 3 Uterine rupture with complete opening of the uterine wall at the site of the previous transverse scar.

uterine incision. The frequency of uterine rupture is approximately $1 \%$ with previous low-segment transverse cesarean section during labor and approximately 4 to $9 \%$ with a vertical or T-shaped section. ${ }^{1}$ The subsequent pregnancy outcome after conservative management of uterine rupture has only been studied in several small case series, among which the prevalence of recurrence is in a wide range of approximately 0 to $33 \%$. $^{2,3}$

Uterine rupture typically occurs during active labor in the third trimester, but it can also occur earlier in pregnancy. ${ }^{4}$ In the literature, most cases are associated with labor procedures performed using agents for late termination. ${ }^{5,6}$ Reports in which uterine rupture occurred spontaneously in the early trimesters are quite rare, ${ }^{4}$ and the reported causes include uterine placenta percreta with or without a scarred uterus, $4,7,8$ rudimentary horn pregnancy, ${ }^{9,10}$ coronal resection because of previous ectopic pregnancy, ${ }^{11,12}$ intrauterine anomaly, ${ }^{13}$ and other conditions. ${ }^{14-17}$ In this case, the placenta was attached to the posterior uterine wall, far from the previous incision.

Uterine ruptures that occur during any gestational period may be equally difficult to predict. ${ }^{7,15,17,18}$ The present patient was being evaluated for nonobstetric differential diagnoses related to upper abdominal pain because she presented with less acute signs and symptoms in addition to normal sonographic appearances of the uterus and fetus. The emergency laparotomy was performed 3 hours after admission when her hemodynamically stable condition deteriorated to hypovolemic shock. Intra-abdominal hemorrhage was detected on the second sonography, but neither the uterine rupture nor the extruded fetus was detected by the second sonography or the subsequent first CT scan. This finding suggested that the uterine rupture was latently progressing with bleeding and that the rupture was completed with fetal extrusion after the first CT scan was performed. Although the dynamic imaging CT scan requested by the surgical team to determine the incision region for the laparotomy contributed to the diagnosis of uterine rupture, we should have aborted the preoperative investigation and taken the patient to the operating room immediately after the hemoperitoneum was found on the first scan to prevent maternal collapse. We also experienced difficulty in obtaining a timely correct diagnosis of uterine rupture to prevent acute deterioration of the patient's condition.

This patient had short interpregnancy (IP) intervals after a previous cesarean delivery, which is considered as a risk factor for uterine rupture during vaginal birth after cesarean (VBAC) because of the insufficient healing time for the uterine scar. ${ }^{19,20}$ In 2004, the American College of Obstetricians and Gynecologists stated that women who attempt VBAC with IP intervals less than 24 months have a 2-fold to 3-fold higher risk of uterine rupture than women who attempt VBAC more than 24 months after their last delivery, ${ }^{21}$ a finding that is relevant in this case. In a similar case, uterine rupture occurred at 18 weeks' gestation, 4 months after a low-segment transverse cesarean section. ${ }^{17}$ In that report, the uterus ruptured immediately after intercourse, and the Nassar et al suggested that the rupture may be associated with short IP interval, uterine contraction induced by sexual intercourse with nipple and clitoral stimulation leading to orgasm, and the prostaglandin D within the seminal plasma.

In her previous pregnancy, our patient had undergone cervical conization because of cervical cancer 3 years previously; the conization resulted in short cervical length and the need for a Shirodkar cerclage. According to the obstetric information from the hospital where the cesarean section was performed, the massive bleeding on admission originated from the cervical laceration, where pulsatile active bleeding was observed to an extent that required blood transfusion. Although it was not recorded whether bleeding through the cervical canal was also observed, she consequently underwent emergency cesarean delivery under the diagnosis of placental abruption because a retroplacental hematoma was found and no scar was detected on intraoperative ocular inspection of the inside and outside surfaces of the uterine wall. In her complicated history of previous pregnancies, there was no well-known risk factor for predicting subsequent uterine rupture except for the short IP intervals after cesarean delivery. However, one challenging hypothesis is that the uterine wall may have already suffered from unrecognized damage in addition to the unexpected cervical laceration before the cesarean section.

Fox et $\mathrm{al}^{22}$ investigated the labor outcomes of 69 patients who had a Shirodkar cerclage placed during pregnancy and removed before labor. They reported five cases (7.2\%) of cervical laceration and two cases $(2.9 \%)$ of uterine rupture during labor in unscarred uteruses; these findings suggest high susceptibility to uterine injury with the use of Shirodkar cerclage, even after its removal. Interestingly, among the two cases of uterine rupture reported in the literature, one patient received a Shirodkar cerclage with a history of cryotherapy, similar to our case. The unrecognized damage because of the Shirodkar cerclage in addition to the cesarean scar may have resulted in uterine rupture early on in the subsequent 
pregnancy. Furthermore, investigations are needed to clarify the correlation between Shirodkar cerclage and uterine rupture in subsequent pregnancies.

\section{Conclusion}

We reported a case of uterine rupture in the second trimester of pregnancy in a patient with a history of cesarean delivery after removal of a Shirodkar cerclage. Although little information is available about the relationship between prior Shirodkar cerclage and subsequent pregnancy outcomes, a history of Shirodkar cerclage might increase the risk of subsequent uterine rupture in women who have undergone cesarean section. Further investigation of this issue is required to confirm our findings.

\section{References}

1 American College of Obstetricians and Gynecologists. ACOG practice bulletin. Vaginal birth after previous cesarean delivery. Number 5, July 1999 (replaces practice bulletin number 2, October 1998). Clinical management guidelines for obstetriciangynecologists. Int J Gynaecol Obstet 1999;66(2):197-204

2 Reyes-Ceja L, Cabrera R, Insfran E, Herrera-Lasso F. Pregnancy following previous uterine rupture. Study of 19 patients. Obstet Gynecol 1969;34(3):387-389

3 Ritchie EH. Pregnancy after rupture of the pregnant uterus. A report of 36 pregnancies and a study of cases reported since 1932.J Obstet Gynaecol Br Commonw 1971;78(7):642-648

4 Vaknin Z, Maymon R, Mendlovic S, Barel O, Herman A, Sherman D. Clinical, sonographic, and epidemiologic features of second- and early third-trimester spontaneous antepartum uterine rupture: a cohort study. Prenat Diagn 2008;28(6):478-484

5 Levrant SG, Wingate M. Midtrimester uterine rupture. A case report. J Reprod Med 1996;41(3):186-190

6 Chapman SJ, Crispens M, Owen J, Savage K. Complications of midtrimester pregnancy termination: the effect of prior cesarean delivery. Am J Obstet Gynecol 1996;175(4 Pt 1):889-892

7 Hornemann A, Bohlmann MK, Diedrich K, et al. Spontaneous uterine rupture at the $21 \mathrm{st}$ week of gestation caused by placenta percreta. Arch Gynecol Obstet 2011;284(4):875-878

8 Honig A, Rieger L, Thanner F, Eck M, Sutterlin M, Dietl J. Placenta percreta with subsequent uterine rupture at 15 weeks of gestation after two previous cesarean sections. J Obstet Gynaecol Res 2005; 31(5):439-443

9 Kawthalkar AS, Gawande MS, Jain SH, Joshi SA, Ghike SD, Bhalerao AV. Rare case of live birth in a ruptured rudimentary horn pregnancy. J Obstet Gynaecol Res 2011;37(8):1169-1172

10 Lauritsen MP, Larsen E, Johnsen M. Second trimester pregnancy in a rudimentary uterine horn. Acta Obstet Gynecol Scand 2010; 89(8):1111-1112

11 Langton J, Fishwick K, Kumar B, Nwosu EC. Spontaneous rupture of an unscarred gravid uterus at 32 weeks gestation. Hum Reprod 1997;12(9):2066-2067

12 Arbab F, Boulieu D, Bied V, Payan F, Lornage J, Guérin JF. Uterine rupture in first or second trimester of pregnancy after in-vitro fertilization and embryo transfer. Hum Reprod 1996;11(5): 1120-1122

13 Damiani GR, Gaetani M, Landi S, et al. Uterine rupture in a nulliparous woman with septate uterus of the second trimester pregnancy and review in literature. Int J Surg Case Rep 2013;4(3): 259-261

14 Singhal SR, Gupta A, Nanda S. Spontaneous asymptomatic uterine scar dehiscence at 20 weeks of gestation as a result of endomyometritis. Arch Gynecol Obstet 2009;280(4):689-690

15 Matsuo K, Shimoya K, Shinkai T, et al. Uterine rupture of cesarean scar related to spontaneous abortion in the first trimester. J Obstet Gynaecol Res 2004;30(1):34-36

16 Retzke JD, Book NM, Stempel LE. Spontaneous second-trimester uterine rupture in the absence of known risk factors: a case report. J Reprod Med 2009;54(8):525-528

17 Nassar A, Usta I, Finianos A, Kaspar H. Spontaneous uterine rupture following intercourse. Acta Obstet Gynecol Scand 2004;83(1): 114-115

18 Endres LK, Barnhart K. Spontaneous second trimester uterine rupture after classical cesarean. Obstet Gynecol 2000;96(5 Pt 2): 806-808

19 Shipp TD, Zelop CM, Repke JT, Cohen A, Lieberman E. Interdelivery interval and risk of symptomatic uterine rupture. Obstet Gynecol 2001;97(2):175-177

20 Stamilio DM, DeFranco E, Paré E, et al. Short interpregnancy interval: risk of uterine rupture and complications of vaginal birth after cesarean delivery. Obstet Gynecol 2007;110(5): 1075-1082

21 American College of Obstetricians and Gynecologists. ACOG Practice Bulletin \#54: vaginal birth after previous cesarean. Obstet Gynecol 2004;104(1):203-212

22 Fox NS, Rebarber A, Bender S, Saltzman DH. Labor outcomes after Shirodkar cerclage. J Reprod Med 2009;54(6):361-365 